\title{
SPLITTINGS OF SURFACES
}

\author{
RICHARD K. SKORA
}

Let $F$ be a compact 2-manifold without boundary and with Euler characteristic $\chi(F)<0$. Only for convenience endow $F$ with a fixed hyperbolic structure, i.e., a discrete, faithful representation of the fundamental group $\pi_{1} F$ into the space of isometries of hyperbolic 2-space. Teichmüller space, $\mathscr{T}(F)$, is the space of all hyperbolic structures on $F$ divided out by conjugation. W. P. Thurston [Th1] showed that $\mathscr{T}(F)$ admits a compactification as a ball of dimension $-3 \chi(F)$. There is a natural identification of the interior of the ball with $\mathscr{T}(F)$ and the boundary of the ball with the space of projective measured geodesic laminations on $F$ (defined below).

J. W. Morgan and P. B. Shalen [MS1, Mo] considered a more general problem. Let $\Gamma$ be a finitely generated, nonvirtually Abelian group and let $\mathscr{D}_{n}=\mathscr{D}\left(\Gamma, \operatorname{Isom}\left(H^{n}\right)\right)$ be the space of discrete, faithful representations of $\Gamma$ into the group of isometries of hyperbolic $n$-space divided out by conjugation. They showed that $\mathscr{D}_{n}$ admits a compactification $\widehat{\mathscr{D}}_{n}$ where each point of $\widehat{\mathscr{D}}_{n}-\mathscr{D}_{n}$ corresponds to a small action of $\Gamma$ on an R-tree. When $\Gamma=\pi_{1} F$ and $n=2$, they too show that their boundary $\widehat{\mathscr{D}}_{n}-\mathscr{D}_{n}$ is homeomorphic to the space of projective measured geodesic laminations on $F$.

An R-tree is a metric space $(T, d)$, such that any two distinct points are joined by a unique arc and every arc is isometric to an interval in $\mathbf{R}$. It is understood that if a group acts on an $\mathbf{R}$-tree, then it acts by isometries and there is no invariant, proper subtree. An action is small if the stabilizer of each arc does not contain a free group of rank two.

The above results motivate studying small actions of $\Gamma$ on $\mathbf{R}$ -

Received by the editors June 28, 1989 and, in revised form, December 20, 1989.

1980 Mathematics Subject Classification (1985 Revision). Primary 30F25; Secondary $32 \mathrm{G} 15,22 \mathrm{E} 40,20 \mathrm{~F} 32$.

Key words and phrases. Surface, hyperbolic structure, lamination, tree.

Supported in part by a SUNY Research Development Grant and by a National Science Foundation Postdoctoral Research Fellowship. 
trees. When $\Gamma=\pi_{1} F$, we completely characterize small actions and answer a question from [Sh]. Recall that the only small subgroups of $\pi_{1} F$ are cyclic groups. Here is our main theorem (definitions follow immediately). It has a generalization for a compact 2-manifold with boundary.

Theorem. Let $\pi_{1} F \times T \rightarrow T$ be an action on an $\mathbf{R}$-tree. Then $\pi_{1} F \times T \rightarrow T$ is dual to a measured geodesic lamination if and only if the stabilizer of each arc is cyclic.

A geodesic lamination $\mathscr{L}$ is a closed subset of $F$, such that each path component is a simple geodesic. A geodesic lamination is discrete if it is a finite union of simple closed geodesics. Say an arc in $F$ is transverse to $\mathscr{L}$ if its endpoints lie on the complement of $\mathscr{L}$ and it is transverse locally. A transverse measure $\mu$ is a function from the set of transverse arcs to the set $[0,+\infty)$, such that (i) $\mu\left(\gamma+\gamma^{\prime}\right)=\mu(\gamma)+\mu\left(\gamma^{\prime}\right)$; and (ii) $\mu(\gamma)=\mu\left(\gamma^{\prime}\right)$, whenever $\gamma, \gamma^{\prime}$ differ by a 1-parameter family of transverse arcs. One may think of a transverse measure on a discrete geodesic lamination as simply an assignment of weights to each geodesic. The set of measured discrete geodesic laminations is dense in the space of measured geodesic laminations [Th1].

Let $\mathbf{H}^{2} \rightarrow F$ be the universal covering. Given a measured geodesic lamination $(\mathscr{L}, \mu)$ in $F$ its preimage in $\mathbf{H}^{2}$ is $(\widetilde{\mathscr{L}}, \tilde{\mu})$. Say the action $\pi_{1} F \times T \rightarrow T$ is dual to $(\mathscr{L}, \mu)$ if there is an equivariant, locally constant map $p: \mathbf{H}^{2}-\widetilde{\mathscr{L}} \rightarrow T$, such that $\tilde{\mu}(\gamma)=d(p(\gamma(0)), p(\gamma(1)))$, for every transverse arc $\gamma:[0,1] \rightarrow$ $\mathbf{H}^{2}$ meeting each path component of $\mathscr{L}$ at most once.

Morgan and J.-P. Otal [MO] proved the above theorem under an additional geometric hypothesis (cp. [Sk]). And H. Gillet and $P$. B. Shalen [GS] proved it under the additional hypothesis that the action has rank equal to 1 or 2 .

The techniques of J. Stallings [MS1] prove the theorem when the R-tree is a simplicial tree. In this case it has the following interpretation. The Bass-Serre theory [Se] implies that the action on the simplicial tree gives a splitting of $\pi_{1} F$, e.g., a free product with amalgamation or HNN-extension. And the lamination which will be discrete is called a splitting of $F$.

\section{Degenerations of HYPeRBolic STRUCTURES ON SURFACES}

Let $\rho \in \mathscr{D}_{n}=\mathscr{D}\left(\pi_{1} F, \operatorname{Isom}\left(H^{n}\right)\right)$. Define its length function $l: \pi_{1} F \rightarrow \mathbf{R}$ by $l(g)=\inf _{x \in \mathbf{H}^{n}} d(x, \rho(g)(x))$. Now form the 
projective space $\mathscr{P}=[0,+\infty)^{\pi_{1} F}-0 / \sim$. One gets a map $\Theta$ : $\mathscr{D}_{n} \rightarrow \mathscr{P}$ by sending a representation to its projectivized length function.

Let $\pi_{1} F \times T \rightarrow T$ be an action on an $\mathbf{R}$-tree. Define its length function $l: \pi_{1} F \rightarrow \mathbf{R}$ by $l(g)=\inf _{x \in T} d(x, g(x))$. A small action of $\pi_{1} F$ is determined by its length function [CM]. The space of projective classes of small length functions on trees $\mathscr{S} \mathscr{L F}\left(\pi_{1} F\right)$ is the image in $\mathscr{P}$ of all small actions on R-trees.

Morgan and Shalen showed that the closure $\overline{\Theta\left(\mathscr{D}_{n}\right)}$ is compact and that $\overline{\Theta\left(\mathscr{D}_{n}\right)}-\Theta\left(\mathscr{D}_{n}\right)$ is a subset of $\mathscr{S} \mathscr{L} \mathscr{F}\left(\pi_{1} F\right)$. This leads to the compactification $\widehat{\mathscr{D}}_{n}$. Identify $\mathscr{D}_{n}$ with its image in $\widehat{\mathscr{D}}_{n}$ and let $\partial \mathscr{D}_{n}=\widehat{\mathscr{D}}_{n}-\mathscr{D}_{n}$.

Finally given a measured geodesic lamination $(\mathscr{L}, \mu)$ it has a length function $l: \pi_{1} F \rightarrow R$, where $l(g)$ is the transverse measure of the geodesic representative of $g$. Again the measured geodesic lamination is determined by its length function [Th1, PH]. The space of projective measured geodesic laminations $\mathscr{P} \mathscr{M L}(F)$ is the image in $\mathscr{P}$ of all measured geodesic laminations.

Thurston showed $\mathscr{P} \mathscr{L} \mathscr{L}(F)=\partial \mathscr{D}_{2}$. By construction $\partial \mathscr{D}_{2} \subseteq$ $\partial \mathscr{D}_{n} \subseteq \mathscr{S} \mathscr{L} \mathscr{F}\left(\pi_{1} F\right)$. And the main theorem implies

$$
\mathscr{S} \mathscr{L F}\left(\pi_{1} F\right) \subseteq \mathscr{P} \mathscr{M L}(F) .
$$

Theorem. For all $n \geq 2, \partial \mathscr{D}_{n}=\mathscr{P} \mathscr{M} \mathscr{L}(F)$.

The above theorem in the cases $n=2,3$ was first proved by Thurston [Th2].

\section{SMALl actions of SURface groups on R-TReeS}

The main theorem also has applications to the study of surface group actions on R-trees. From [Ha] or [MS2] $\mathscr{P} \mathscr{M} \mathscr{L}(F) \subseteq$ $\mathscr{S} \mathscr{L F}\left(\pi_{1} F\right)$. Again by the main theorem $\mathscr{S} \mathscr{L F}\left(\pi_{1} F\right) \subseteq$ $\mathscr{P} \mathscr{M} \mathscr{L}(F)$. The following answers a question of $\mathrm{M}$. Culler and J. W. Morgan [CM] in the case the group is $\pi_{1} F$.

Theorem. $\mathscr{S} \mathscr{L} \mathscr{F}\left(\pi_{1} F\right)=\mathscr{P} \mathscr{M L}(F)$.

Since every measured geodesic lamination is approximable by a measured discrete geodesic lamination, the above theorem tells us that every small action of $\pi_{1} F$ on an $\mathbf{R}$-tree is approximable by a small action on a simplicial tree. It is an open question whether every (small) action of a finitely generated group on an R-tree is aproximable by a (small) action on a simplicial tree [Sh]. 
Finally we may deduce two finiteness results. Fix a small action $\pi_{1} F \times T \rightarrow T$ and the dual measured geodesic lamination $(\mathscr{L}, \mu)$. A vertex of $T$ is a point $x$, where $T-\{x\}$ has more than two connected components. The vertices of $T$ correspond to the connected components of $\mathbf{H}^{2}-\widetilde{\mathscr{L}}$. An area calculation shows the number of orbits of vertices is no greater than $-2 \chi(F)$.

The rank of the action is the dimension of $G \otimes \mathbf{Q}$ as a vector space over $\mathbf{Q}$, where $G$ is the subgroup of $\mathbf{R}$ equal to $\langle l(g)\rangle_{g \in \pi_{1} F}$. Again referring back to the lamination, one sees that the rank is no greater than one plus the dimension of $\mathscr{P} \mathscr{M L}(F)$ which is $-3 \chi(F)$.

\section{SKetch OF THE PROOF OF THE MAIN THEOREM}

Suppose that $\pi_{1} F \times T \rightarrow T$ is dual to a measured geodesic lamination. Then the stabilizer of each arc in $T$ is contained in the fundamental group of a path component of the lamination which is cyclic.

Now conversely suppose the action has cyclic arc stabilizers. The starting point is a theorem of A. Hatcher [Ha] or Morgan and Otal [MO]. They prove that there is an action on an R-tree $\pi_{1} F \times R \rightarrow R$ and an equivariant morphism $\phi: R \rightarrow T$, such that $\pi_{1} F \times R \rightarrow R$ is dual to a measured geodesic lamination $(\mathscr{L}, \mu)$ on $F$.

A morphism from $R$ to $T$ is a map $\phi: R \rightarrow T$, such that for each arc $[x, y]$ in $R$ there is an $\operatorname{arc}[x, z] \subseteq[x, y]$, such that $\phi \mid[x, z]$ is an isometry. The morphism $\phi$ folds at a point $x \in R$ if there are arcs $[x, y]$ and $\left[x, y^{\prime}\right]$ such that $[x, y] \cap\left[x, y^{\prime}\right]=$ $\{x\} ; \phi \mid[x, y]$ and $\phi \mid\left[x, y^{\prime}\right]$ are embeddings; and $\phi([x, y])=$ $\phi\left(\left[x, y^{\prime}\right]\right)$. A morphism either is a monomorphism or folds at some point. Thus it suffices to show that $\phi$ does not fold.

We will prove the theorem by contradiction. Suppose $\phi$ folds at $x$. Let $[x, y]$ and $\left[x, y^{\prime}\right]$ be as in the definition of fold. We may suppose $x$ is a vertex.

Suppose $R$ is a simplicial tree. Then $\mathscr{L}$ is a discrete geodesic lamination and up to rechoosing we may suppose $[x, y],\left[x, y^{\prime}\right]$ have infinite cyclic stabilizers $\langle g\rangle,\left\langle g^{\prime}\right\rangle$, respectively. It is easy to see from the geometry of $\mathscr{L}$ that $\langle g\rangle,\left\langle g^{\prime}\right\rangle$ are conjugate, but $\left\langle g, g^{\prime}\right\rangle$ is free of rank two. Therefore the stabilizer of $\phi([x, y])=$ $\phi\left(\left[x, y^{\prime}\right]\right)$ contains this free group of rank two which is a contradiction. 
Now for $R$ a general $\mathbf{R}$-tree the proof proceeds by studying $(\mathscr{L}, \mu)$ more carefully. An important tool is a train track. A train track is a smooth subgraph $\tau$ of $F$, such that the double of each component of $F-\tau$ along its smooth frontier has negative Euler characteristic. An important combinatorial property of $\tau$ is that every smoothly immersed curve $\gamma$ in $\tau$ is determined by the lift to $\mathbf{H}^{2}$ of its initial and terminal points [Th1, $\left.\mathrm{PH}\right]$. In particular, every smoothly immersed loop is nontrivial. Say that a lamination is carried by a train track $\tau$ if there is a map $f: F \rightarrow F$ fixed on $\tau$ and homotopic to the identity, such that $f$ composed with each smooth curve in $\mathscr{L}$ is a smooth immersion. Every geodesic lamination is carried by a train track [Th1, PH].

The second tool is an interval exchange map. Let $\alpha$ be transverse to $\mathscr{L}$ with lift $\tilde{\alpha}$ in $\widetilde{L}$, such that the image of $\tilde{\alpha}$ in $R$ is $[x, y]$ and $\mu(\alpha)=d(x, y)$. Fix an orientation and transverse orientation on $\alpha$ and let $I$ be an interval of length $\mu(\alpha)$. Then parallel translation of $\alpha$ along $\mathscr{L}$ determines an interval exchange map $A: I \rightarrow I$. If we identify $[x, y]$ with $I$, then there are a finite number of elements $g_{1}, \ldots \in \pi_{1} F$ which permute the subarcs of $[x, y]$ exactly the same way $A$ permutes the subintervals of $I$. It follows that words of length $n$ in $g_{1}, \ldots$ permute the subarcs of $[x, y]$ exactly the same way $A^{n}$ permutes the subintervals of $I$.

So corresponding to both $[x, y],\left[x, y^{\prime}\right]$ are interval exchange maps $A, A^{\prime}$ respectively. Or equivalently, there are group elements $g_{1}, \ldots$ and $g_{1}^{\prime}, \ldots$ which permute subarcs of $[x, y]$ and $\left[x, y^{\prime}\right]$, respectively. Let $\tau$ carry $\mathscr{L}$. Up to passing to a finite fold covering and rechoosing $[x, y]$ and $\left[x, y^{\prime}\right]$ we may suppose that distinct positive words in $g_{1}, \ldots, g_{1}^{\prime}, \ldots$ are represented by distinct smoothly immersed loops in $\tau$.

Since $[x, y]$ and $\left[x, y^{\prime}\right]$ have identical images in $T$, we should consider the way $g_{1}, \ldots, g_{1}^{\prime}, \ldots$ permute the subarcs of $\phi([x, y])$. For any $z \in \phi([x, y])$, let $\mathscr{C}_{n}$ be the set of sequences $\left\langle h_{1}, \ldots, h_{n}\right\rangle$, such that $h_{i} \in\left\{g_{1}, \ldots, g_{1}^{\prime}, \ldots\right\}$ and $h_{i} \circ \ldots \circ$ $h_{1}(z) \in \phi([x, y])$, for all $i$. The set $\mathscr{C}_{n}$ grows exponentially with $n$.

However, $A, A^{\prime}$ are defined by finitely many translations. So their $n$-fold compositions are defined by a certain number of translations which grows polynomially with $n$. Therefore $\mathscr{C}_{n}(z)$ grows polynomially with $n$. Now one may argue for all but at most 
countably many $z$ and for large enough $n$, there are distinct positive words $w, v, u$ in the elements $g_{1}, \ldots, g_{1}^{\prime}, \ldots$ which agree on some subarc of $\phi([x, y])$. By our choice of $\tau$ the words $w$, $v, u$ represent distinct elements of $\pi_{1} F$. Thus $w^{-1} v, w^{-1} u$ fix an arc. Finally, by choosing $w, v, u$ carefully the elements $w^{-1} v, w^{-1} u$ will generate a free group of rank two. This is a contradiction.

\section{REFERENCES}

[CM] M. Culler and J. W. Morgan, Group actions on R-trees, Proc. London Math. Soc. 55 (1987), 571-604.

[GS] H. Gillet and P. B. Shalen, Dendrology of groups in low Q-ranks, J. Differential Geometry (to appear).

[Ha] A. Hatcher, Measured lamination spaces for surfaces from the topological viewpoint, Topology Appl. 30 (1988), 63-88.

[Mo] J. W. Morgan, Group actions on trees and the compactification of the spaces of classes of $S O(n, 1)$-representations, Topology 25 (1986), 1-34.

[MO] J. W. Morgan and J.-P. Otal, Relative growth rates of closed geodesics on a surface under varying hyperbolic structures, preprint.

[MS1] J. W. Morgan and P. B. Shalen, Degenerations of hyperbolic structures, I: Valuations, trees and surfaces, Ann. of Math. 120 (1984), 401-476.

[MS2] _ Surface groups acting on R-trees, preprint.

[PH] R.C. Penner and J. L. Harer, Combinatorics of train tracks, preprint.

[Se] J.-P. Serre, Trees, Springer-Verlag, New York, 1980.

[Sh] P. B. Shalen, Dendrology of groups: An introduction, in Essays in Group Theory (S. M. Gersten ed.), Mathematical Sciences Research Institute Publications, \#8, Springer-Verlag, New York, 1987, pp. 265-319.

[Sk] R. K. Skora, Geometric actions of surface groups on $\Lambda$-trees, Comm. Math. (to appear).

[Th1] W. P. Thurston, Geometry and topology of 3-manifolds, preprint.

[Th2] __, Hyperbolic structures on 3-manifolds, II: Surface groups and 3-manifolds which fiber over the circle, preprint.

Department of Mathematics, State University of New York, Stony BROOK, NEW YORK 11794-3651

Department of Mathematics, Columbia University, New York, New YORK 10027 